\title{
Size-Dependent Adhesion Strength of a Single Viscoelastic Fiber
}

\author{
Shaohua Chen $\cdot$ Guang Xu $\cdot$ Aikah Soh
}

Received: 26 April 2009/ Accepted: 19 October 2009/Published online: 6 November 2009

(C) Springer Science+Business Media, LLC 2009

\begin{abstract}
Nano-fibrillar adhesives can adhere strongly to surfaces as a gecko does. The size of each fiber has significant effects on the adhesion enhancement, especially on rough surfaces. In the present study, we report the size effects on the normal and shear strength of adhesion for a single viscoelastic fiber. It is found that there exists a limited region of the critical sizes under which the interfacial normal or tangential tractions uniformly attain the theoretical adhesion strength. The region for a viscoelastic fiber under tension with similar material constants to a gecko's spatula is $135-255 \mathrm{~nm}$ and that under torque is $26.5-52 \mathrm{~nm}$. This finding is significant for the development of artificial biomimetic attachment systems.
\end{abstract}

Keywords Fibrillar structure · Viscoelasticity ·

Size effect $\cdot$ Adhesion strength

\section{Introduction}

Geckos have evolved elaborate adhesive structures which allow them to climb on vertical walls and ceilings against their body weight. The mechanical mechanism is due to the van der Waals force $[1,2]$ between a contacting surface and geckos' hierarchical micro-scale/nano-scale foot hairs. Many adhesion and contact mechanics models for the micro- and nano-fibrillar interfaces [3-10] have been

S. Chen $(\bowtie) \cdot G$. Xu

LNM, Institute of Mechanics, Chinese Academy of Sciences, 100190 Beijing, China

e-mail: chenshaohua72@hotmail.com

A. Soh

Department of Mechanical Engineering, The University of Hong Kong, Hong Kong, China developed, in which it is found that the adhesion strength of the contact interface is affected significantly by the size of fibers and there exists a critical size under which the interfacial adhesion strength saturates to be the theoretical one. This gives some insights to the phenomenon that the larger the weight of an animal, the finer the setae will be. Recently, synthetic fibrillar adhesives [11-14] have been fabricated using vertically aligned multi-walled carbon nanotubes or polymer, to amplify adhesion of a flat contact, in which each fiber has a cylindrical shape different from that of the spatula in geckos. Friction properties and normal adhesion strength are measured experimentally and both are found to be enhanced in contrast to a smooth surface. Due to the viscoelastic properties, especially for the polymer pillars, the preload in the experiments should be relaxed for sometime [13]. Furthermore, the natural materials in attachment structures also exhibit viscoelastic properties $[15,16]$. This leads us to the following question: what is the effect of viscoelasticity on the nano-pillar's adhesion?

In the present study, the size effect of a single cylindrical fiber on the adhesion strength is studied with the help of numerical calculations and the fiber is considered to be viscoelastic under both tensile and torque loadings, respectively. It is found that the normal/shear adhesion strength will be significantly improved with a decreasing radius of the fiber at a fixed tensile/torque loading rate. A critical value of the viscoelastic fiber radius exists, at which the interfacial adhesion strength will be saturated to be the theoretical strength. Furthermore, critical values vary along with different loading rates and tend to be two finite constants when the loading rate is smaller than a threshold or larger than another one. This finding is significant for the development of artificial biomimetic attachment systems. 


\section{Simulation Model}

The simulating model is shown in Fig. 1, in which a viscoelastic fiber of radius $R$ contacts a rigid half-space due to the molecular interactions. In the same manner as Gao et al. [4], imperfect contact between the fiber and substrate is assumed such that the radius of the actual contact area is $a=k R, 0<k<1$, as shown in Fig. 1; the outer rim $k R<r<R$ represents flaws or regions of poor adhesion. Thus, the contact interface becomes a circumferentially cracked region. During the calculation, it is found that the aspect ratio of the viscoelastic fiber does not show a significant effect on the results. On the other hand, Yao and Gao [6] showed that the length of fiber would be helpful in hierarchical structures to improve the apparent work of adhesion on the next level, but the critical radius of a single fiber was not influenced by its aspect ratio in the first level. Thus, we choose the aspect ratio to be $3: 4$ in the present study.

In order to model the viscoelastic property of fibers, we choose the shear modulus $\mu$ varying with the time $t$ according to an ordinary linear viscoelastic law, $\mu(t)=$ $\mu_{\infty}+\mu_{\text {neq }} \exp \left(-t / t_{\mathrm{s}}\right)$, where $t_{\mathrm{s}}$ is the relaxation time of shear modulus. One should be noted that other viscoelastic laws can also be used to find the effect of viscoelasticity on adhesion. The bulk modulus is assumed to be a constant and Poisson's ratio be 0.25 . According to the snapshot of a moving gecko [17], we take the typical relaxation time as $t_{\mathrm{s}}=2$ ms. Both the relaxed shear moduli $\mu_{\infty}$ and $\mu_{\text {neq }}$ are taken as $0.8 \mathrm{GPa}$, which results in a varying Young's modulus during the whole relaxation process as shown in Fig. 2. The instantaneous Young's modulus $E_{0}$ is $3.43 \mathrm{GPa}$ and the relaxed one is $2 \mathrm{GPa}$ when the relaxation time is over $10 \mathrm{~ms}$, which is identical to the Young's modulus of a gecko's spatula used in [4].

In order to simulate adhesive interactions between two surfaces, cohesive surface element is used, whose

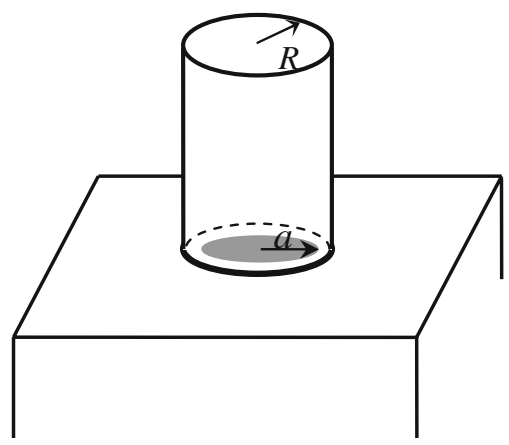

Fig. 1 Schematic of a viscoelastic nano-fiber of radius $R$ in adhesive contact with a rigid half-space due to the interfacial molecular interactions. The shadow area with radius $a$ denotes the actual contact one

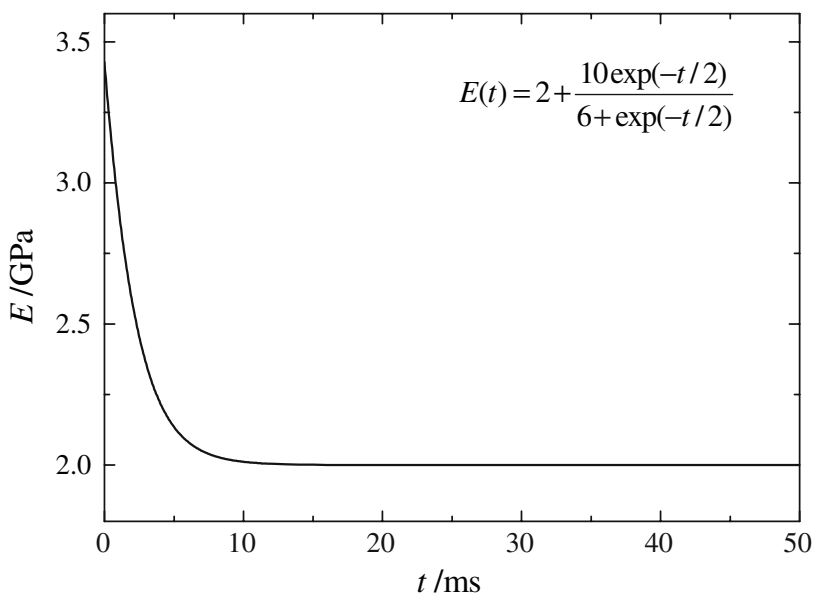

Fig. 2 Variation of the viscoelastic Young's modulus $E$ along with the relaxation time $t$

constitutive relation is specified in terms of a relation between the interfacial traction and separation across the contact interface. A general-purpose finite element code Tahoe [18] supports a number of traction-separation laws including the $\mathrm{Xu}-\mathrm{Needleman}$ [19] and the TvergaardHutchinson [20] laws. We adopt the latter with the schematic in Fig. 3, because it preserves the van der Waals energy regardless of the loading orientation. The tractionseparation law can be written as

$\varphi(\lambda)=\left\{\begin{array}{l}\varphi_{\max } \lambda / \Lambda_{1},\left(\lambda<\Lambda_{1}\right) \\ \varphi_{\max },\left(\Lambda_{1}<\lambda<\Lambda_{2}\right) \\ \varphi_{\max }(1-\lambda) /\left(1-\Lambda_{2}\right),\left(\Lambda_{2}<\lambda<1\right)\end{array}\right.$

where $\varphi(\lambda)$ is the instantaneous traction, $\lambda$ is a non-dimensional parameter describing the instantaneous separation, $\lambda=\sqrt{\left(\Delta_{\mathrm{n}} / \delta_{\mathrm{cn}}\right)^{2}+\left(\Delta_{\mathrm{t}} / \delta_{\mathrm{ct}}\right)^{2}}, \Delta_{\mathrm{n}}$ denotes the instantaneous normal separation and the instantaneous tangential separation $\Delta_{\mathrm{t}}$ is composed of two parts $\Delta_{\mathrm{t} 1}$ and $\Delta_{\mathrm{t} 2}$ in two axial

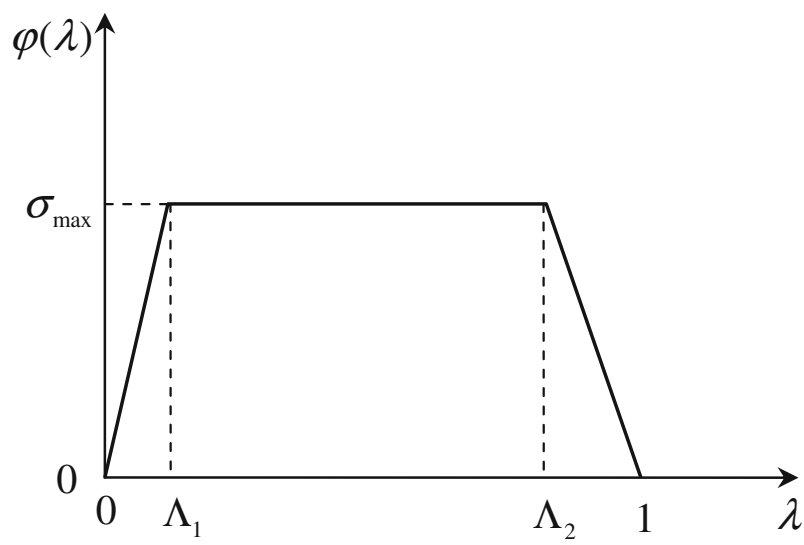

Fig. 3 Schematic of the relation between the interface traction $\varphi(\lambda)$ and the non-dimensional displacement $\lambda$ in Tvergaard-Hutchinson cohesive law 
directions with $\Delta_{t}=\sqrt{\Delta_{t 1}^{2}+\Delta_{t 2}^{2}} . \delta_{\mathrm{cn}}$ and $\delta_{\mathrm{ct}}$ are the corresponding critical separations. $\varphi_{\max }$ is the maximum value of traction that the interface could support. The work of adhesion of the interface is assumed to be a constant as $\Delta \gamma=0.5\left(1+\Lambda_{2}-\Lambda_{1}\right) \varphi_{\max } \delta_{\mathrm{cn}}$. The values of $\Lambda_{1}$ and $\Lambda_{2}$ does not show significant influence on the calculating results [21], we choose $\Lambda_{1} \approx 0, \Lambda_{2} \approx 1$ in the present paper. The work of adhesion is assumed to be $0.01 \mathrm{~J} / \mathrm{m}^{2}$ in the same manner as in [4] and $\varphi_{\max }$ is taken as $20 \mathrm{MPa}$ in the tension case, which results in the critical normal separation $\delta_{\mathrm{cn}}=$ $0.5 \mathrm{~nm}$. The theoretically tangential strength [22] of the contact interface is taken as $\mu^{*} / 43$, where $\mu^{*}$ is a compound shear modulus, $1 / \mu^{*}=1 / \mu_{1}+1 / \mu_{2}, \mu_{1}$ and $\mu_{2}$ are the shear moduli of the two solids forming the interface, respectively. Due to the rigid substrate, $\mu^{*}$ is identical to that of the fiber, which results in $\varphi_{\max }=18.6 \mathrm{MPa}$ and $\delta_{\mathrm{ct}} \approx 0.54 \mathrm{~nm}$ in the torque case.

\section{Size Effect in Tension Case}

In the tension case, a uniform displacement is added on the above surface of the viscoelastic fiber according to $u=\alpha t$, where $\alpha$ is the loading rate with units $\mathrm{nm} / \mathrm{ms}$ and $t$ is the incremental time varying from $0 \mathrm{~ms}$ to the moment of pulloff. Different loading rate corresponds to different failure time, which will yield different instantaneous Young's modulus at the moment of pull-off.

The computational results clearly show that the traction distribution in the contact region becomes more uniform as the radius of viscoelastic fiber is reduced. For a fixed loading rate as an example, $\alpha=0.2 \mathrm{~nm} / \mathrm{ms}$, the traction distributions, $T_{\mathrm{n}}$, within the contact region just before the moment of pull-off are plotted in Fig. 4 for cases with three different fiber sizes, which is very similar to the elastic results in Gao et al. [4]. It is observed that the stress concentration near the contact edge vanishes despite of the crack-like flaw around the contact region, when the radius of viscoelastic fiber is below a critical size. Comparing to the model for an elastic fiber in Gao et al. [4], one can infer that an instantaneous traction distribution in the present
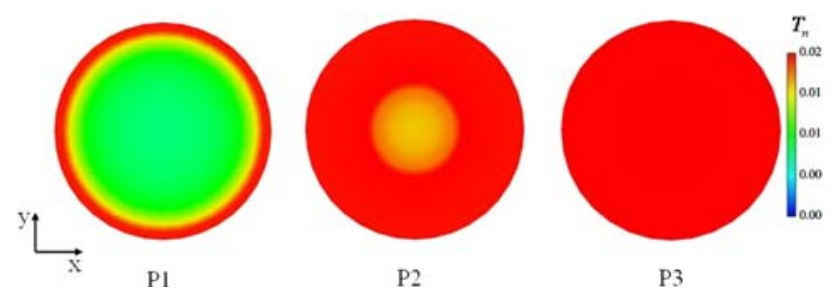

Fig. 4 The normal traction distribution within the contact region becomes more uniform as the size of the fiber is reduced. The radii of $\mathrm{P} 1, \mathrm{P} 2$, and $\mathrm{P} 3$ are 800,200 , and $10 \mathrm{~nm}$, respectively. An arbitrary scale is used here

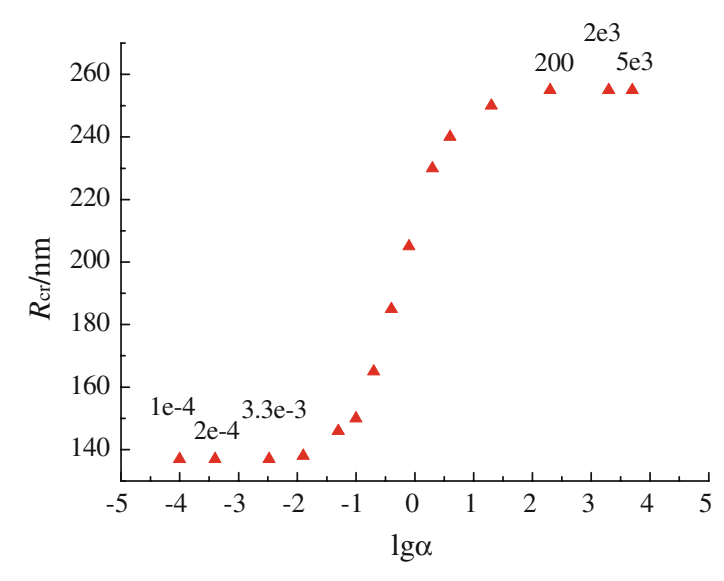

Fig. 5 The critical size varies along with the tension rates. The data near each point denotes the true loading rate with units $\mathrm{nm} / \mathrm{ms}$

viscoelastic case should correspond to a counterpart in the elastic one.

Due to the viscoelastic property of the fiber, different loading rates should have impacts on the critical size. Figure 5 shows the critical size varying along with the loading rates. It is found that there exist two limitations at a smaller or larger loading rate, at which the critical size tends to be a finite constant and does not depend on the loading rates any longer. The region of the critical size for strength saturation at different loading rates is nearly from 135 to $255 \mathrm{~nm}$. Interestingly, the radius of the gecko's spatula is typically around $100-250 \mathrm{~nm}$ [1]. The viscoelastic analysis in the present work suggests that a viscoelastic fiber with nano-scales may achieve optimization of adhesive strength in tolerance of possible contact flaws, and adhesives made from such kinds of fibers may also tolerant the pulling velocities due to the critical size limitations. But one should be noted that the spatula in geckos' foot is similar to a finite film subject to peeling forces. Whether the concept of flaw tolerance for a viscoelastic fiber under tension adapts to the peeling case of geckos' spatula is a future work.

\section{Size Effect in Torque Case}

The interfacial adhesion shear strength is also investigated for a viscoelastic fiber in contact with a rigid substrate under pure torque. In the torque case, a rotational angle is uniformly added on the above surface of the viscoelastic fiber through $\omega=\beta t$, where $\beta$ denotes the rate of angular velocity with units $\mathrm{rad} / \mathrm{ms}$ and $t$ is the incremental time varying from $0 \mathrm{~ms}$ to the moment of shear-off.

The computational results clearly indicate that the tangential tractions tend to be uniformly distributed as the radius of viscoelastic fiber decreases. For a fixed rate of 


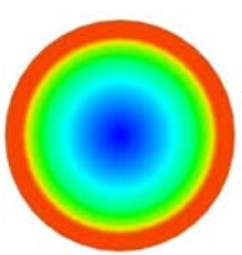

P1

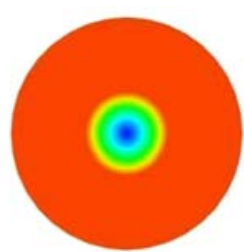

$\mathrm{P} 2$

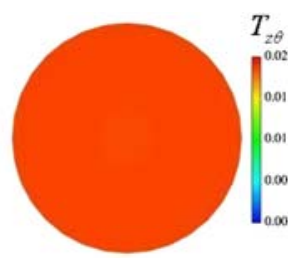

P3
Fig. 6 The tangential traction distribution within the contact region becomes more uniform when the size of the fiber is reduced. The radii of P1, P2, and P3 are 600, 100, $20 \mathrm{~nm}$, respectively. An arbitrary scale is used here

angular velocity, $\beta=0.04 \mathrm{rad} / \mathrm{ms}$, the shear traction distributions just before the moment of shear-off are plotted in Fig. 6 for three cases with different representative fiber sizes. It is observed that the tangential stress concentration near the contact edge vanishes when the radius of the fiber is below a critical one.

The effect of the loading rate of angular velocity on the critical size is found to have a similar manner as that in the tension case, the critical size for tangential strength saturation is affected significantly by the loading rates as shown in Fig. 7. Two limitations exist at smaller or larger loading rates and tend to be finite constants. The critical size under different torque loading rates varies between 26.5 and $52 \mathrm{~nm}$. These data are much smaller than those under tension, which implies a viscoelastic fiber with cylindrical shape and nanometer length scale should be flaw-sensitivity under torque behavior and can detach easily from solid surfaces with a very small torque.

\section{Discussions}

Size effects of adhesion of a single viscoelastic fiber under tension and torque are studied using finite element method

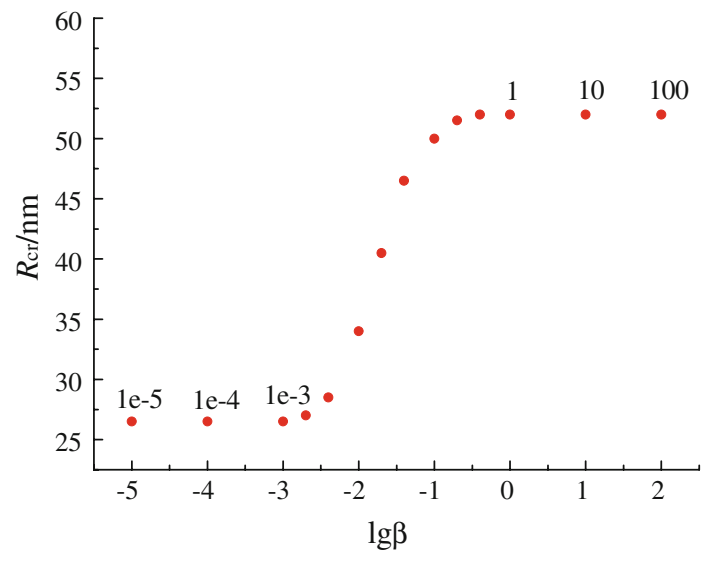

Fig. 7 The critical size varies along with the torque rates. The data near each point denotes the true rate of angular velocity with units $\mathrm{rad} / \mathrm{ms}$ with specialized cohesive surface elements in the present paper. The critical sizes are determined, below which the normal or tangential tractions become uniform and equal to the corresponding theoretical strength. It is found that the critical size varies from 135 to $255 \mathrm{~nm}$ under tension force and from 26.5 to $52 \mathrm{~nm}$ under torque for a viscoelastic fiber with cylindrical shape and material constants similar to that of a gecko's spatula. The data imply to some degree that nano-fibrillar structure adhesives composed of such kinds of fibers could attain strong adhesion and realize reversible adhesion easily through alternate tension and torque behaviors. The findings may be valuable in shedding light on the design of biomimetic adhesives. Whether the same manner exists in the detachment behavior of geckos' spatula can not be inferred because the spatula on geckos' foot is subjected to a peeling force.

In contrast to the theoretical predictions for an elastic fiber under tension or torque $[4,23]$, one can find that the critical size of a viscoelastic fiber to achieve optimization of adhesive strength under tension is consistent with the theoretical result [4]. However, the simulating result under torque has a little bit large difference from the theoretical one [23]. Some of the possible causes of the deviation could be the dependence of the interface decohesion on the fracture criterion. In the theoretical model, Griffith energy release rate criterion is adopted, while TvergaardHutchinson cohesive law is used in the present numerical simulation. In addition, a finite circular adhesion interface with a circumferentially cracked region under pure torque can not be strictly identical to an infinite mode III crack case.

Certainly many factors should be considered in future efforts about size effects of viscoelastic fibers, such as the roughness of natural surfaces, capillary forces, contact shapes, fiber fracture, fiber condensation, etc., most of which have been included in adhesion design maps [24], but not accounted for in the present work so far.

Acknowledgments The authors acknowledge the National Science Foundation (10972220, 10732050, and 10721202), KJCX2-YW-M04 of CAS Innovation Program and Start Fund for Returning Overseas person.

\section{References}

1. Autumn, K., Liang, Y.C., Hsieh, S.T., Zesch, W., Chan, W.P., Kenny, T.W., Fearing, R., Full, R.J.: Adhesion forces of a single gecko foot-hair. Nature 405, 681-685 (2000)

2. Autumn, K., Sitti, M., Liang, Y.C., Peattie, A.M., Hansen, W.R., Sponberg, S., Kenny, T.W., Fearing, R., Israelachvili, J.N., Full, R.J.: Evidence for van der Walls adhesion in gecko setae. Proc. Natl Acad. Sci. USA 99, 12252-12256 (2002)

3. Glassmaker, N.J., Jagota, A., Hui, C.Y., Kim, J.: Design of biomimetic fibrillar interface: 1. Making contact. J. R. Soc. Interface 1, 23-33 (2004) 
4. Gao, H., Wang, X., Yao, H., Gorb, S., Arzt, E.: Mechanics of hierarchical adhesion structures of gecko. Mech. Mater. 37, 275285 (2005)

5. Hui, C.Y., Glassmaker, N.J., Tang, T., Jagota, A.: Design of biomimetic fibrillar interface: 2. Mechanics of enhanced adhesion. J. R. Soc. Interface 1, 35-48 (2004)

6. Yao, H., Gao, H.: Bio-inspired mechanics of robust and releasable adhesion on rough surface. J. Mech. Phys. Solids 54, 1120 1146 (2006)

7. Chen, S., Gao, H.: Bio-inspired mechanics of reversible adhesion: orientation-dependent adhesion strength for non-slipping adhesive contact with transversely isotropic elastic materials. J. Mech. Phys. Solids 55, 1001-1015 (2007)

8. Arzt, E., Gorb, S., Spolenak, R.: From micro to nano contacts in biological attachment devices. Proc. Natl Acad. Sci. USA 100, 10603-10606 (2003)

9. Chen, S., Soh, A.: Tuning the geometrical parameters of biomimetic fibrillar structures to enhance adhesion. J. R. Soc Interface 5, 373-382 (2008)

10. Persson, B.N.J.: Nanoadhesion. Wear 254, 832-834 (2003)

11. Geim, A.K., Dubonos, S.V., Grigorieva, I.V., Novoselov, K.S., Zhukov, A.A., Shapoval, S.: Microfabricated adhesive mimicking gecko foot-hair. Nat. Mater. 2, 461-463 (2003)

12. Kim, S., Sitti, M.: Biologically inspired polymer microfibers with spatulate tips as repeatable fibrillar adhesives. Appl. Phys. Lett. 89, 261911 (2006)

13. Kim, S., Aksak, B., Sitti, M.: Enhanced friction of elastomer microfiber adhesives with spatulate tips. Appl. Phys. Lett. 91, 221913 (2007)
14. Maeno, Y., Nakayama, Y.: Geckolike high shear strength by carbon nanotube fiber adhesive. Appl. Phys. Lett. 94, 012103 (2009)

15. Arzt, E.: Biological and artificial attachment devices: lessons for materials scientists from flies and geckos. Mater. Sci. Eng. C 26, 1245-1250 (2006)

16. Persson, B.N.J.: On the mechanism of adhesion in biological systems. J. Chem. Phys. 118(16), 7614-7621 (2003)

17. Russell, A.P.: A contribution to the functional analysis of the foot of the Tokay, Gekko gecko (Reptilia, Gekkonidae). J. Zool. Lond. 176, 437-476 (1975)

18. FEM code developed by Dr. Patrick Klein of Sandia National Laboratory, http://tahoe.cs.sandia.gov

19. Xu, X.P., Needleman, A.: Numerical simulations of fast crack growth in brittle solids. J. Mech. Phys. Solids 42, 1397-1434 (1994)

20. Tvergaard, V., Hutchinson, J.W.: Effect of strain-dependent cohesive zone model on predictions of crack growth resistance. Int. J. Solids Struct. 33, 3297-3308 (1996)

21. Yao, H.: Mechanics of robust and releasable adhesion in biology. $\mathrm{PhD}$ dissertation, Max-Planck Institute for Metals Research, Stuttgart (2006)

22. Carpick, R.W., Agrait, N., Ogletree, D.F., Salmeron, M.: Variation of the interfacial shear strength and adhesion of a nanometer sized contact. Langmuir 12, 3334-3340 (1996)

23. Chen, S., Xu, G., Soh, A.K.: Robust nanoadhesion under torque. Tribol. Lett. 29, 235-239 (2008)

24. Spolenak, S., Gorb, S., Arzt, E.: Adhesion design maps for bioinspired attachment systems. Acta Biomater. 1, 5-13 (2005) 